\title{
Mapping the Stellar Halo with the H3 Spectroscopic Survey
}

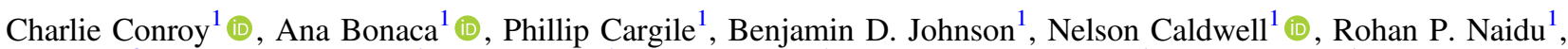 \\ Dennis Zaritsky ${ }^{2}$ (D), Daniel Fabricant ${ }^{1}$, Sean Moran ${ }^{1}$, Jaehyon Rhee ${ }^{1}$, Andrew Szentgyorgyi ${ }^{1}$, Perry Berlind ${ }^{1}$, Michael L. Calkins ${ }^{1}$, \\ ShiAnne Kattner ${ }^{2}$, and Chun Ly ${ }^{2}$ \\ ${ }^{1}$ Center for Astrophysics | Harvard \& Smithsonian, Cambridge, MA 02138, USA \\ ${ }^{2}$ Steward Observatory, University of Arizona, 933 North Cherry Avenue, Tucson, AZ 85721, USA \\ Received 2019 May 14; revised 2019 July 12; accepted 2019 August 4; published 2019 September 25
}

\begin{abstract}
Modern theories of galaxy formation predict that the Galactic stellar halo was hierarchically assembled from the accretion and disruption of smaller systems. This hierarchical assembly is expected to produce a high degree of structure in the combined phase and chemistry space; this structure should provide a relatively direct probe of the accretion history of our Galaxy. Revealing this structure requires precise 3D positions (including distances), 3D velocities, and chemistry for large samples of stars. The Gaia satellite is delivering proper motions and parallaxes for $>1$ billion stars to $G \approx 20$. However, radial velocities and metallicities will only be available to $G \approx 15$, which is insufficient to probe the outer stellar halo $(\gtrsim 10 \mathrm{kpc})$. Moreover, parallaxes will not be precise enough to deliver high-quality distances for stars beyond $\sim 10 \mathrm{kpc}$. Identifying accreted systems throughout the stellar halo therefore requires a large ground-based spectroscopic survey to complement Gaia. Here we provide an overview of the H3 Stellar Spectroscopic Survey, which will deliver precise stellar parameters and spectrophotometric distances for $\approx 200,000$ stars to $r=18$. Spectra are obtained with the Hectochelle instrument at the MMT, which is configured for the H3 Survey to deliver resolution $R \approx 23,000$ spectra covering the wavelength range $5150-5300 \AA$. The survey is optimized for stellar halo science and therefore focuses on high Galactic latitude fields $\left(|b|>30^{\circ}\right)$, sparsely sampling 15,000 sq. degrees. Targets are selected on the basis of Gaia parallaxes, enabling very efficient selection of bona fide halo stars. The survey began in the fall of 2017 and has collected 88,000 spectra to-date. All of the data, including the derived stellar parameters, will eventually be made publicly available via the survey website: h3survey.rc.fas.harvard.edu.
\end{abstract}

Key words: Galaxy: halo - Galaxy: kinematics and dynamics

\section{Introduction and Motivation}

Simulations of hierarchical structure formation predict that the stellar halo contains an extraordinary amount of structure in the high dimensional space $(6 \mathrm{D}$ phase plus $\mathrm{N}$ dimensional abundances plus stellar ages) occupied by stars (e.g., Johnston et al. 1996; Helmi \& White 1999; Bullock \& Johnston 2005; Cooper et al. 2010). Thankfully, historical memory in the Galactic halo stretches back billions of years due to long relaxation times; one of the principal goals of Galactic Archeology is to uncover this history and use it to trace the assembly of our Galaxy (e.g., Helmi 2008). Learning this assembly history impacts many fields of astronomy, including galaxy formation, the cosmological evolution of structure, and studies of the nature of dark matter.

The distribution of stars in energy and angular momentum, e.g., $E-L_{Z}$ space, encodes the accretion history of galaxies, as demonstrated in Figure 1. In this figure we show three simulated stellar halos with both "early" and "late" accretion histories (from the Aquarius simulations; Cooper et al. 2010; Lowing et al. 2015). The distribution of stars in these model halos in energy and angular momentum varies systematically with accretion history. The measurement of the phase space structure of the Galactic stellar halo should therefore place novel constraints on the Galaxy's assembly history.

The Gaia mission (Perryman et al. 2001) is propelling a revolution in our understanding of the stellar halo (e.g., Belokurov et al. 2018; Helmi et al. 2018). Gaia is delivering parallaxes and proper motions for $>1$ billion stars to $G \approx 20$. In DR2 (Gaia Collaboration et al. 2018), radial velocities (RVs) were measured for stars brighter than $G \approx 12$; the end-of-mission projection predicts that RVs with precision $5-10 \mathrm{~km} \mathrm{~s}^{-1}$ will be available to $G \approx 15$. This leaves a significant gap, of $\approx 5 \mathrm{mag}$, where Gaia will provide constraints on parallaxes and proper motions for stars lacking RVs. This gap must be filled by large ground-based spectroscopic surveys.

The need for spectroscopic information is motivated in Figure 2. Here we show a simulated stellar halo from the Aquarius simulation halo A2. In the upper panels we show all of the stars in gray and highlight in orange the stars belonging to a single progenitor. The upper left panel shows a sky projection in the northern hemisphere. It is clear that debris from a single progenitor is spread across the sky, necessitating large area surveys. The right panel shows a projection of phase space: energy and the $z$-component of the angular momentum, $E-L_{Z}$, in which many structures are clearly visible. The middle panel shows pseudo $E-L_{Z}$ space, in which no RVs are available and so we have assumed $\mathrm{RV}=0.0 \mathrm{~km} \mathrm{~s}^{-1}$. While some of the largest structures are still visible, most of the structure is washed away. The bottom panel shows $E-L_{Z}$ space color-coded by stellar metallicity.

Clearly, a spectroscopic survey that can deliver RVs and metallicities for stars fainter than $G \approx 15$, would be highly complementary to the Gaia spectroscopic data. The faintest dwarfs have internal velocity dispersions of order a few $\mathrm{km} \mathrm{s}^{-1}$ (Simon \& Geha 2007), so one would need RVs with a precision of $\lesssim 1 \mathrm{~km} \mathrm{~s}^{-1}$. The bottom panel of Figure 2 suggests that a measurement precision on $[\mathrm{Fe} / \mathrm{H}]$ of $\sigma_{[\mathrm{Fe} / \mathrm{H}]} \sim 0.05 \operatorname{dex}$ should be sufficient to aid in the identification of structure in phase space. 

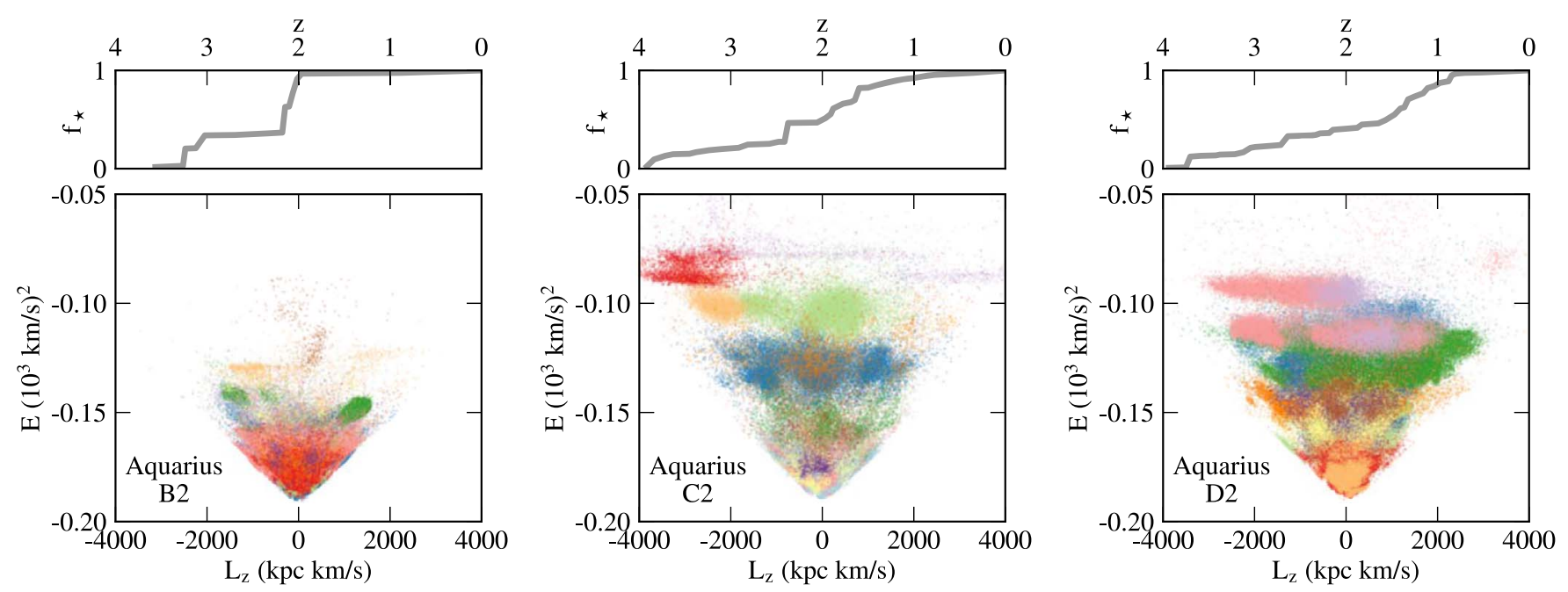

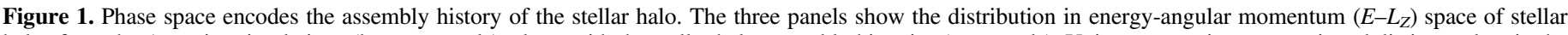

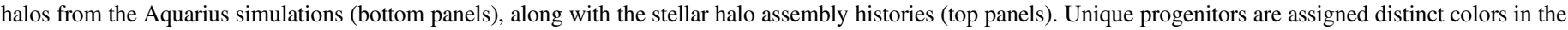

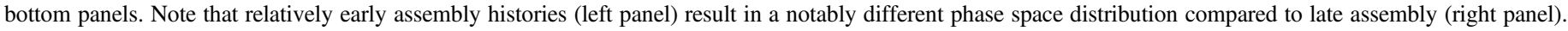

For stars fainter than $G \approx 15$ beyond $\sim 10 \mathrm{kpc}$, Gaia parallaxes are too noisy to determine precision distances. Therefore, spectrophotometric distances (e.g., Burnett \& Binney 2010) are also critically important in order to study the stellar halo. Figure 3 shows the Aquarius A2 halo from Figure 2 as a function of the distance precision. Large uncertainties can have a dramatic effect on the structure in phase space, and uncertainties typically scatter stars along diagonal directions in $E-L_{Z}$ space.

Finally, Figure 4 shows the cumulative distribution of stellar distances in the Rybizki et al. (2018) mock Milky Way catalog for a limiting magnitude of $r=18$ and $r=15$. This mock catalog assumes smooth models for the stellar disk and halo (and is based on the Galaxia synthetic Galaxy). Here we plot stars selected to have a parallax $\pi<0.5$ mas, and to lie within the R.A.-decl. footprint shown in Figure 10 (these details do not affect the main point of Figure 4). It is clear that a relatively bright limit of $\approx 15 \mathrm{mag}$ is insufficient to sample the outer stellar halo.

In summary, a ground-based spectroscopic complement to Gaia that aims to study the entire stellar halo should meet the following requirements: (1) accurate RVs $\left(\sigma_{\mathrm{RV}} \lesssim 1 \mathrm{~km} \mathrm{~s}^{-1}\right)$, spectrophotometric distances (to $\lesssim 10 \%$ ), and metallicities (at a precision of $\lesssim 0.05 \mathrm{dex}$ ); (2) a depth of $\sim 18 \mathrm{mag}$; and (3) cover a large fraction of the sky. These considerations are the motivation behind the $\mathrm{H} 3$ Survey ("Hectochelle in the Halo at High Resolution"), which we describe in detail below. The survey is driven by the following science cases:

1. Identify and characterize disrupting and disrupted dwarf galaxies throughout the Milky Way stellar halo using both phase space information and chemistry. As shown in Figures 1 and 2, phase space is rich with substructure that reflects the accretion and disruption of many dwarf galaxies over the history of the Galaxy. Recent work using Gaia DR2 data has identified one, or possibly more, accreted galaxies in the inner halo (e.g., Helmi et al. 2018; Myeong et al. 2018; Fattahi et al. 2019), although the interpretation of discrete structures in phase space is challenging (Jean-Baptiste et al. 2017).

2. Fully characterize the stellar halo in order to understand the accretion history of the Galaxy. Figure 1 demonstrates that "early" versus "late" accretion histories result in qualitatively different distributions of stars in phase space. There are few if any constraints available regarding the accretion history of our Galaxy and the phase space structure offers one of the strongest discriminants (e.g., Deason et al. 2013).

3. Stellar populations in the halo. There are a wide variety of interesting and unusual stellar populations found in the halo including carbon-enhanced metal-poor (CEMP) stars (Lee et al. 2013), high velocity stars, and very metal-poor stars (Frebel \& Norris 2015). These populations offer valuable clues to the origin of the stellar halo and the structure of the Galaxy.

4. Establish the origin of the in situ stellar halo. Recent hydrodynamic simulations and analysis of Gaia DR1 data suggest that there may be an in situ stellar halo (an "inner" halo) formed from scattered disk stars (e.g., Bonaca et al. 2017). Determining the formation mechanism of an in situ stellar halo will provide unique clues to the early Galaxy and will place constraints on major disruptive events in its dynamical history.

5. Measure the outer mass profile of the Galaxy and the shape of the dark matter halo. Analysis of stellar streams has provided novel but often conflicting conclusions regarding the shape and mass profile of the Galaxy. The total mass of the Galaxy is unknown to a factor of two (Wang et al. 2015), which is a major limiting factor when placing the Galaxy in a cosmological context. Various outstanding puzzles, including the "missing satellites" and "too big to fail" problems become more or less severe depending on the mass of the Galaxy's halo (e.g., Bullock \& Boylan-Kolchin 2017). Velocities and distances in the outer stellar halo will enable strong constraints on the mass of the Galaxy's dark halo.

6. Measure the mass of the Large Magellanic Cloud (LMC) through its gravitational effect on the stellar halo. Recent work has demonstrated that the impact of the LMC on the Galaxy can be substantial, both by inducing movement of the Galactic barycenter due to gravitational acceleration (Gómez et al. 2015; Garavito-Camargo et al. 2019) and a more complicated change in stellar (and dark matter) densities, mean velocities, and velocity dispersions 

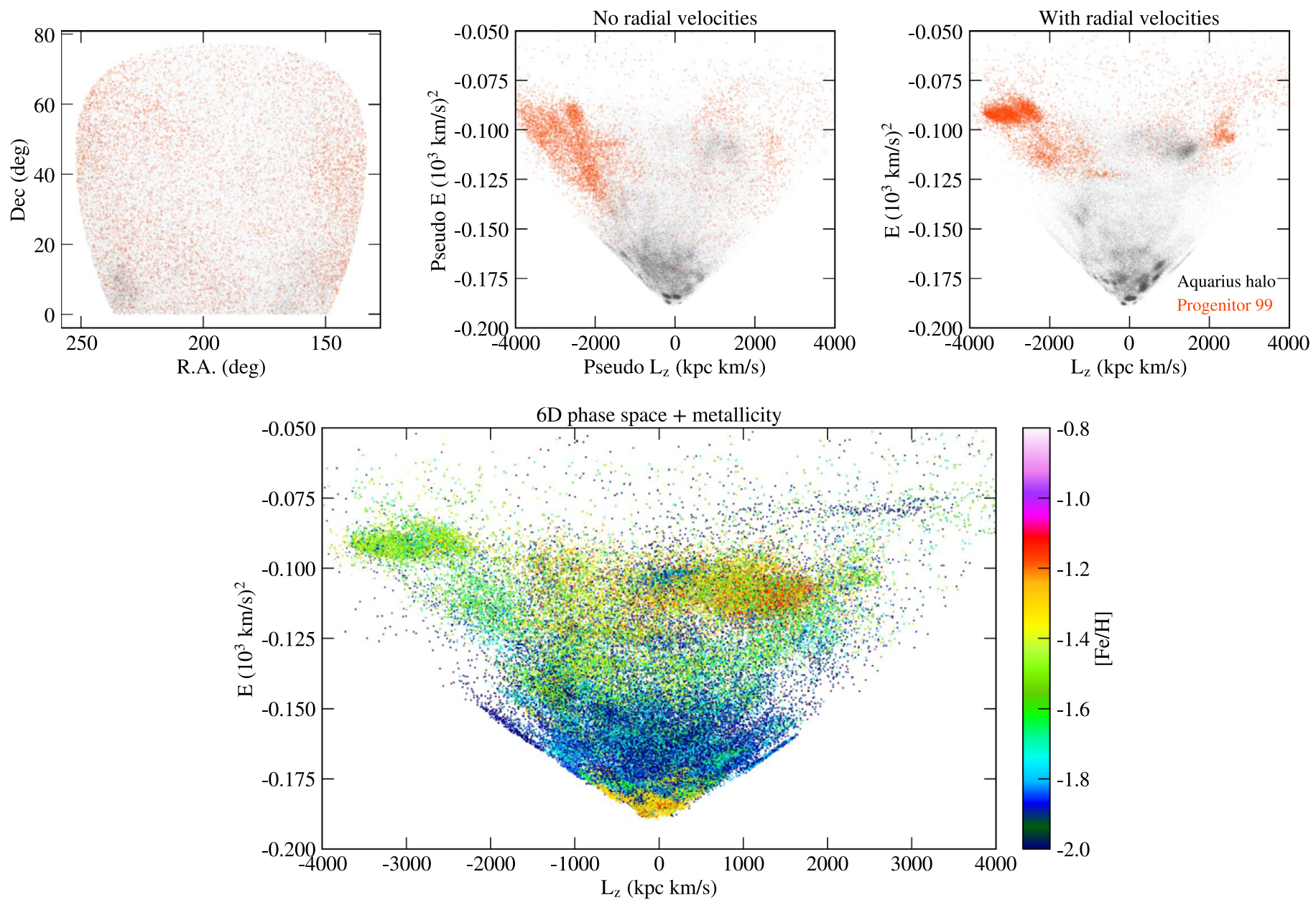

Figure 2. Importance of radial velocities (RVs) and metallicities for revealing structure in phase space. A simulated stellar halo (Aquarius A2) is shown in sky coordinates (upper left), $E-L_{Z}$ space both with (upper right) and without (upper middle) RVs. In the latter case the quantities are estimated assuming RV $=0.0$. The lower panel shows the additional information provided by stellar metallicities. The top set of panels highlights in orange a single massive progenitor. The debris of such a system is spread throughout the sky, necessitating spectroscopic observations over a large fraction of the sky. Note that the addition of radial velocities (upper middle vs. upper right) results in much sharper structures in $E-L_{Z}$ space.

induced in the "wake" of the LMC's orbit (GaravitoCamargo et al. 2019). Belokurov et al. (2019) report a tentative detection of this wake in the direction of the Pisces Overdensity. The reflex motion of the Galactic barycenter can induce a dipole pattern in the RVs across the outer halo as large as $\pm 50 \mathrm{~km} \mathrm{~s}^{-1}$ depending on the mass of the LMC.

This paper is organized as follows. In Section 2 we describe the design of the survey, including the footprint and target selection. Section 3 outlines the observing strategy, data reduction, and stellar parameter pipeline. Survey progress to-date is presented in Section 4 and we place the survey in the context of existing and future spectroscopic surveys in Section 5. A summary is provided in Section 6.

\section{Survey Design}

\subsection{Survey Fields}

As Figure 2 shows, the debris from accreted galaxies is typically spread over large fractions of the sky. A survey aimed at identifying and studying the various components of the stellar halo must therefore cover a large area. Guided by this consideration, our survey strategy is to sparsely sample the entire sky observable from the MMT, while avoiding the Galactic plane. The latter constraint ensures that the fields are not dominated by disk stars. Our survey footprint is therefore the area on the sky encompassing decl. $>-20^{\circ}$ and $|b|>30^{\circ}$, which totals $\approx 15,000$ sq. degrees. Within this footprint we define fields spaced every $3^{\circ}$ in both R.A. and decl. There are 1654 such fields, of which we intend to observe approximately 1000. We do not specifically target, nor avoid, fields with known structures.

\subsection{Input Catalog and Target Selection}

The survey began collecting data in the Fall of 2017, before Gaia DR2 was available, so our input catalog is based on PanSTARRS data release 1 (PS1; Chambers et al. 2016). This primary catalog was matched to WISE (Wright et al. 2010; Cutri 2013), SDSS, 2MASS, and, later, Gaia DR2. The crossmatching was performed using the Large Survey Database framework (Juric 2012) with a matching radius of $<1^{\prime \prime}$.

AGN are identified and removed from the catalog with a WISE color-cut: $0.85<W 1-W 2<2.0$. We require stars to satisfy two Gaia quality cuts: astrometric_excess_noise $<1$ and visibility_periods_used $>5$ (Lindegren et al. 2018). 

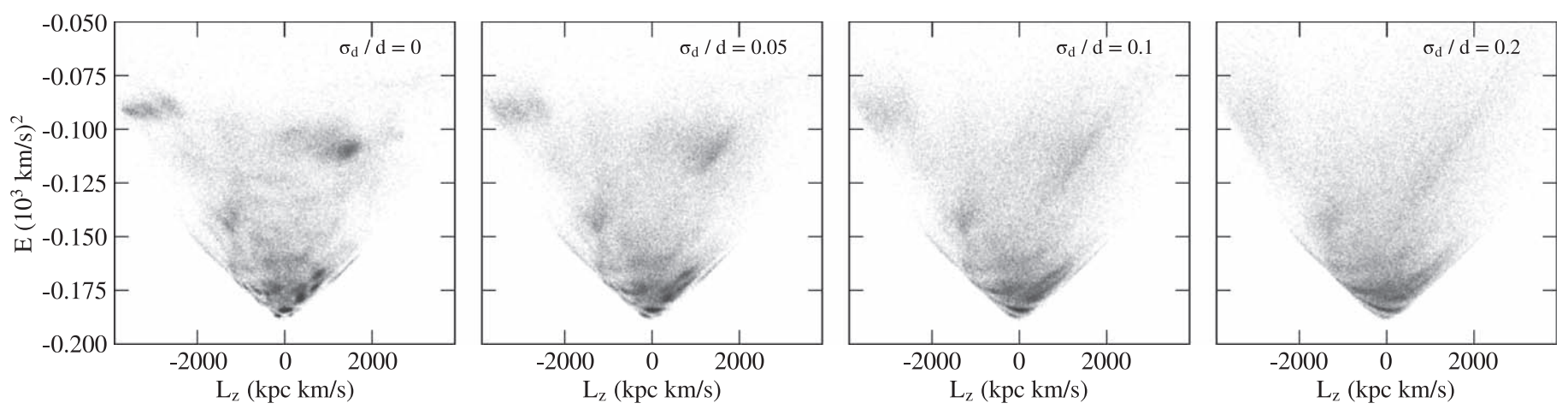

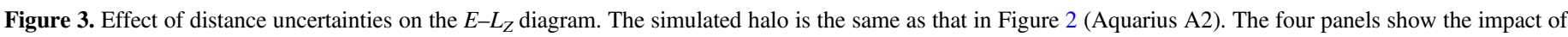
$0 \%, 5 \%, 10 \%$, and $20 \%$ distance uncertainties. Note that many clumps in the leftmost panel turn into diagonal streaks in subsequent panels.

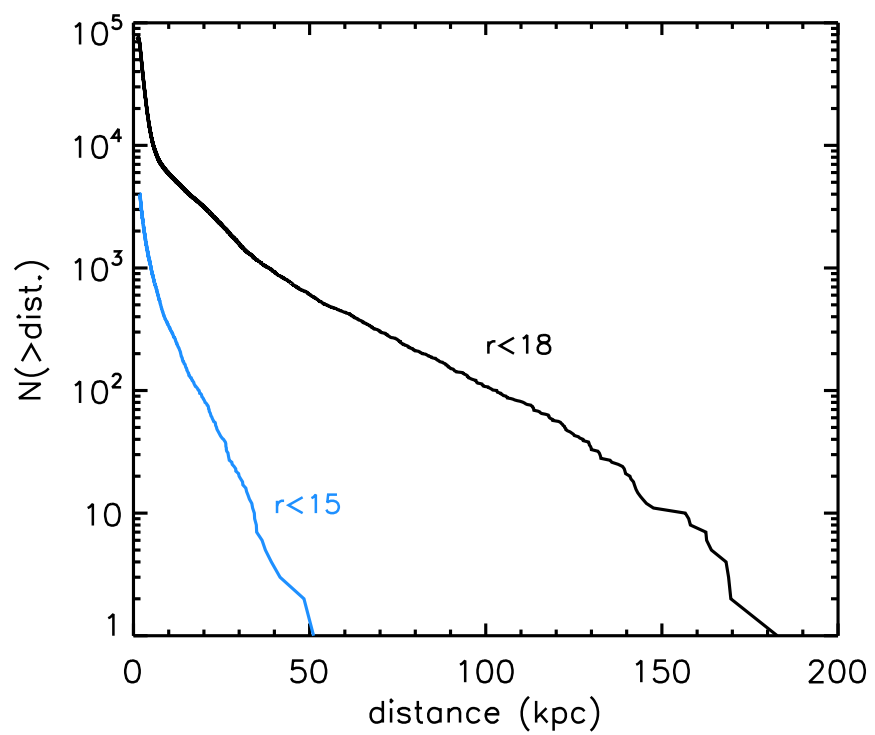

Figure 4. Cumulative distribution of stellar distances in the Rybizki et al. (2018) mock galaxy catalog for stars brighter than a magnitude limit of $r=15$ and $r=18$. Only stars located within the existing $\mathrm{H} 3$ Survey footprint are included (i.e., $|b|>40^{\circ}$ and decl. $>-20^{\circ}$ ). A limiting magnitude of $\approx 15$ is insufficient to sample the outer stellar halo, as even stars at the tip of the red giant branch are too dim to be seen beyond $\approx 50 \mathrm{kpc}$. Note that the Gaia $G$ band is similar to $r$ (to within $\approx 0.05 \mathrm{mag}$ ) for $4000 \mathrm{~K}<T_{\text {eff }}<6500 \mathrm{~K}$.

The over-arching goal of the target selection is to be as simple as possible while optimizing for distant stars. Prior to Gaia DR2 the main sample was defined by the following two requirements: $15<r<18$ and $g-r<1$. The latter is included to filter out the numerous foreground cool dwarfs. A total of 19,301 stars were observed with this selection. After the release of Gaia DR2 the selection switched to a simple parallax cut, with the same magnitude range of $15<r<18$. Specifically, we first employed a parallax selection to ensure that we included all stars that had parallaxes within $2 \sigma$ of being less than 0.5 mas, i.e., $\pi-2 \sigma_{\pi}<0.5$. We recently modified this selection slightly to deliver an even higher halo fraction by adopting $\pi<0.4$ mas as the main sample selection.

There are known systematic issues with the Gaia DR2 parallaxes at the $\lesssim 0.1$ mas level. Lindegren et al. (2018) reported zero-point offsets based on quasars at the 0.03 mas level. However, they recommended against applying an overall zero-point correction to the reported parallaxes, noting substantial small- and large-scale spatial variation in the zeropoints. Subsequent work (e.g., Stassun \& Torres 2018; Leung

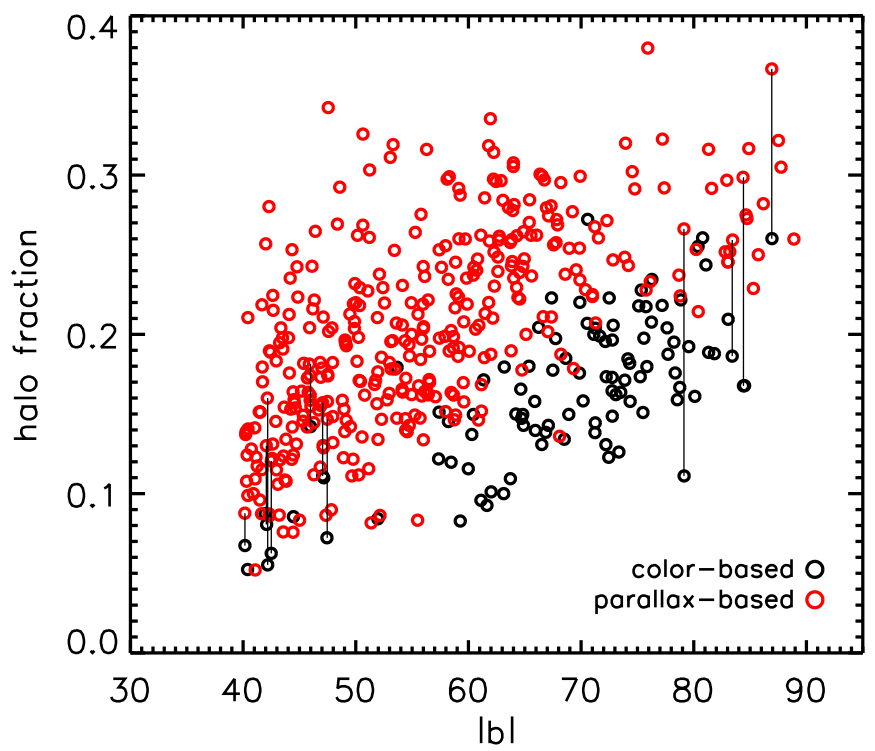

Figure 5. Fraction of stars per field that belong to the kinematic stellar halo, as a function of Galactic latitude. Black symbols show the early survey fields where the target selection was based on color cuts. Red symbols show fields where Gaia parallaxes were used to define the sample. Lines connect the same fields observed with both selections. Notice that our simple Gaia parallax selection results in a much higher fraction of halo stars compared to colorbased selection.

\& Bovy 2019; Schönrich et al. 2019) has confirmed the existence of a global zero-point offset, and has demonstrated that the offset depends on magnitude and color. Owing to the complex nature of the corrections, we have not applied any correction to the DR2 parallaxes at the target selection stage (corrections are applied later when estimating stellar parameters). Hopefully this issue will be resolved in DR3, at which time we will be able to quantify the DR2 zero-point effect on the selection function with mock catalogs.

In addition to the main sample, we also include rare, highvalue targets. We identify $\mathrm{K}$ giants from the input photometric catalogs via a series of optical-IR color cuts described in Conroy et al. (2018). These stars are rare but the purity of the selection is very high $(\approx 85 \%$ probability of being a giant; see Section 4) and they are visible to $>100 \mathrm{kpc}$ at $r=18$. We also select blue horizontal branch stars according to the color cuts presented in Deason et al. (2014), and RR Lyrae from the catalog of Sesar et al. (2017). These three categories of targets are rare, comprising only a few stars per field. They are given 
higher priority than the main sample in assigning fibers to targets.

Two sets of filler targets are included in cases where there are not enough targets in the main and high-value samples to fill the available fibers. The first set of fillers have the same selection as the main sample but are fainter: $18<r<18.5$. The second (lowest priority) fillers have $15<r<18$ and $0.4<\pi<1.0$ mas.

Figure 5 shows the resulting fraction of stars per field that are identified as belonging to the kinematic stellar halo (specifically, $\left|V-V_{\mathrm{LSR}}\right|>220 \mathrm{~km} \mathrm{~s}^{-1}$ ). We compare the halo fraction for the early color-based selection to the later parallax-based color selection. As noted below, 12 fields were observed twice, once for each of the two selections. Solid lines in the figure connect the halo fractions for the same fields observed with the two selection functions. In all cases the halo fraction is substantially higher with the parallax-based selection. The median halo fraction of the parallax-based selection is $20 \%$, with a strong dependence on Galactic latitude (no fields at $30^{\circ}<|b|<40^{\circ}$ have been observed to-date).

\section{Observations and Data Analysis}

\subsection{Observations}

Data are obtained with the medium resolution Hectochelle spectrograph (Szentgyorgyi et al. 2011) on the MMT. Hectochelle employs a robotic fiber positioning system (Fabricant et al. 2005), enabling the placement of 240 fibers over a $1^{\circ}$ diameter field of view. We use the $1101 \mathrm{~mm}^{-1}$ grating and the RV31 order-blocking filter. The data are binned by a factor of three in the wavelength direction and two in the spatial direction. This setup delivers a resolution of $R \approx 23,000$ over the (single order) wavelength range of 5150-5300 $\AA$. This corresponds to a velocity resolution of $\sigma=5.5 \mathrm{~km} \mathrm{~s}^{-1}$ (see also Walker et al. 2015).

In Figure 6 we show a detailed view of four spectral types in order to highlight the atomic and molecular features in the 5150-5300 A wavelength range. We show four spectra: the Solar and Arcturus spectral atlases (from Hinkle et al. 2000), a model M dwarf spectrum (computed from the same grid of models discussed in Section 3.3), and the spectrum of a wellstudied metal-poor giant HD122563, obtained through the UVES archive (Bagnulo et al. 2003). All of these spectra were convolved to a resolution of $R=23,000$. For the Sun we also include the native resolution spectrum in order to assess the line broadening due to the instrument resolution. For the model $\mathrm{M}$ dwarf we have computed a model without the $\mathrm{MgH}$ molecular lines in order to highlight the overwhelming impact of this molecule for metal-rich cool dwarfs in this spectral range. Many of the strongest features are labeled (line identification is adopted from Hinkle et al. 2000).

The survey is being awarded time through the regular TAC processes at the CfA and U. Arizona. The allocations have resulted in approximately 50 nights awarded to the survey per year. Observations are queue scheduled during bright time. The nominal exposure time is $3 \times 10$ minutes, although suboptimal weather conditions occasionally necessitate one or more additional exposures. The exposure time is set by the desire to observe fields as fast as possible while not being dominated by overheads, which are $\approx 15$ minutes per field. This exposure time delivers a signal-to-noise ratio $(\mathrm{S} / \mathrm{N})$ per pixel of $\approx 2$ at the faint limit of the main sample ( $r=18$; see Figure 7 below).
Twilight flat fields are taken most nights and offer an important test of the long-term stability of the derived RV measurements. The twilights are also used for relative fluxing (see below).

The actual number of fibers assigned to science targets varies in the range of 170-200, even when the total number of targets in the field exceeds 400. This is due to fiber "collisions" resulting from the fact that the fibers are robotically moved from the edge of the field inward.

\subsection{Data Reduction Pipeline}

Data are reduced using HSRED v2.1, ${ }^{3}$ an IDL-based pipeline originally developed for Hectospec, but recently extended to handle Hectochelle data as well (HSRED is based on the SDSS idlspec $2 \mathrm{~d}$ algorithms). The code performs wavelength calibration using a fifth-order Legendre fit to the lines identified in ThAr calibration spectra. Flat fielding is performed using a combination of twilight sky flats and dome flats to simultaneously solve for fiber-to-fiber throughput variations and the pixel-to-pixel sensitivity. Dome flats are also used for identifying the traces of each fiber on the chip, which are then extracted using an optimal extraction algorithm. Cosmic rays are removed by an algorithm that identifies outliers in a moving window across all science exposures. Science exposures are sky subtracted by generating an oversampled B-spline model of the sky from the 35-40 designated sky fibers in each observing configuration. Multiple exposures are combined after extraction, using an inverse-variance weighting to optimize $\mathrm{S} / \mathrm{N}$, though simple summed spectra are also generated. Spectra are not interpolated onto a uniform, linear (or log-linear) wavelength grid. The spectra are not flux calibrated. The first 120 fibers are assigned to one CCD while the second 120 are assigned to a second CCD.

The relative system throughput as a function of wavelength is estimated with the following procedure. All available twilight spectra are first divided by a continuum-normalized resolutionmatched spectrum of the Sun. This removes small-scale features not due to system throughput variations. The resulting spectra are then median combined. The resulting stacked spectrum is fit with a 15 th order Chebyshev polynomial. This procedure is performed for each of the two CCDs. This effective throughput curve is then used in the preparation of the data for stellar parameter determination.

\subsection{MINESweeper Stellar Parameter Pipeline}

\subsubsection{Overview}

Stellar parameters are determined using MINESweeper (Cargile et al. 2019). Briefly, the program jointly fits the Hectochelle spectrum and the broadband photometric spectral energy distribution (SED) to a model that is based on MIST (Choi et al. 2016) stellar isochrones. The position along the isochrone is determined by the stellar mass, age, and metallicity. At each point along the isochrone there is a corresponding model SED and high resolution spectrum. We include photometry from Pan-STARRS, SDSS, Gaia, 2MASS, and WISE where available.

The spectral models (and corresponding photometry) are computed from grids of model atmospheres and the spectrum synthesis code SYNTHE (Kurucz 1993). The model atmospheres are computed with the ATLAS12 program (Kurucz

\footnotetext{
https://bitbucket.org/saotdc/hsred
} 


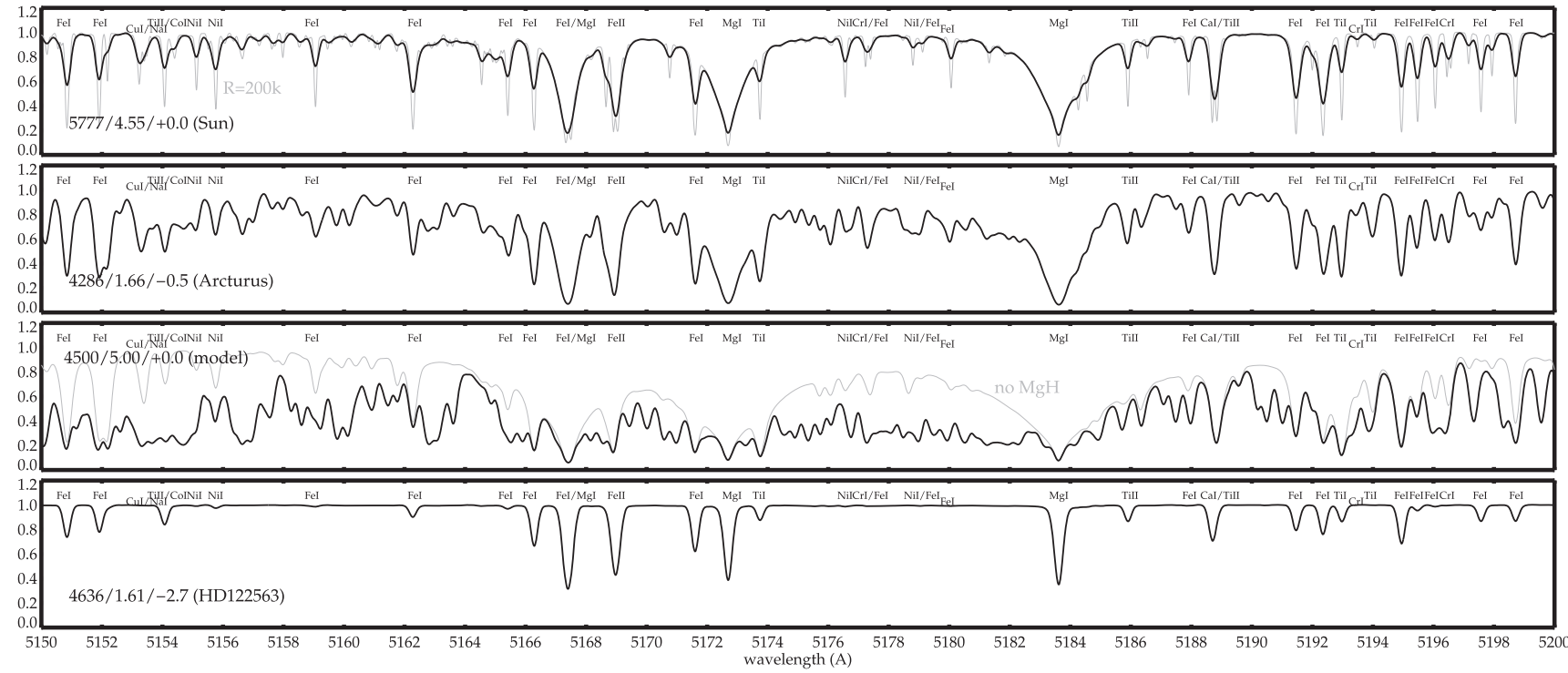

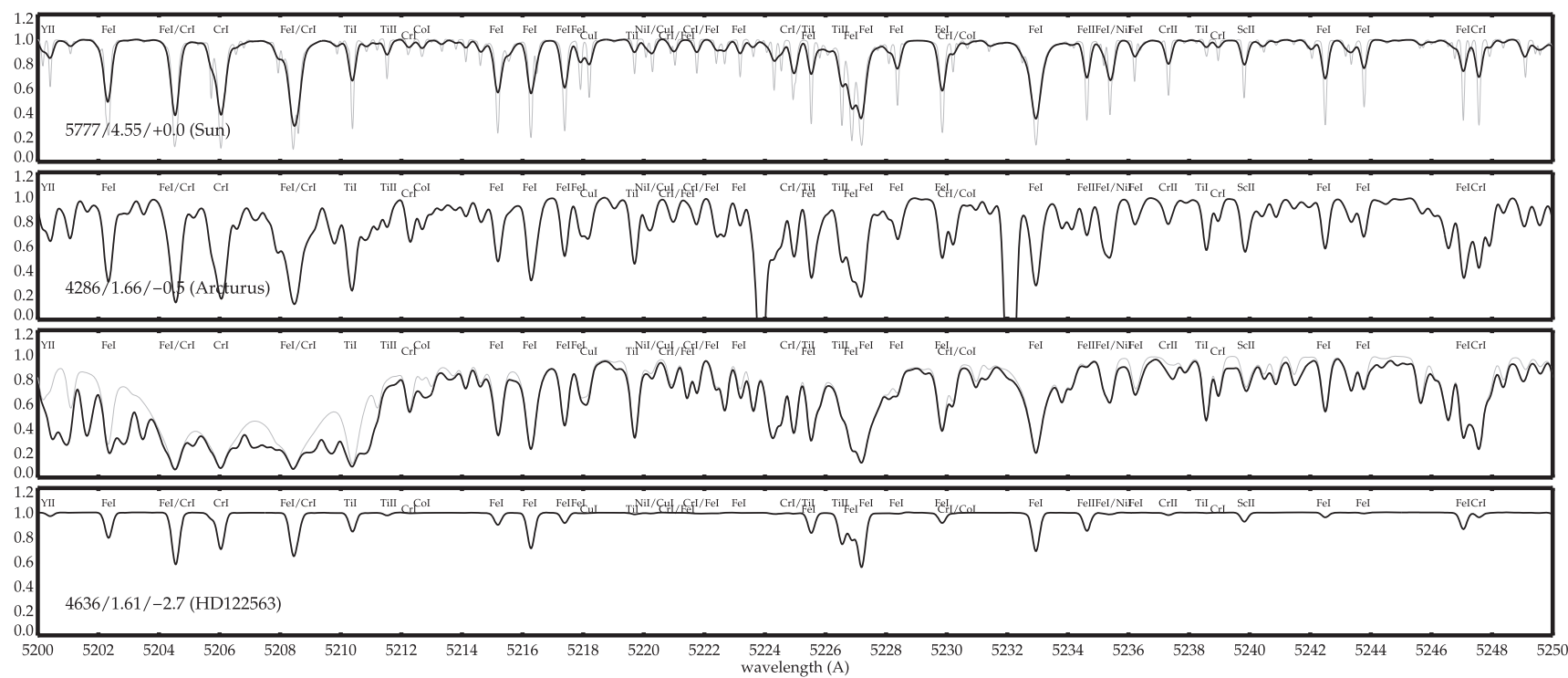

1.2
1.0

Figure 6. Spectra of four example stars in the spectral region covered by the survey. From top to bottom: spectra of the Sun, Arcturus, a model M dwarf, and a metal-poor giant (HD122563). In each panel the stellar parameters are listed as $T_{\text {eff }} / \log g /[\mathrm{Fe} / \mathrm{H}]$. Many of the strongest features are labeled. All spectra are convolved to $R=23,000$. In the top panel the $R \approx 400,000$ solar spectrum is included as a gray line. For the model $\mathrm{M}$ dwarf, we show in gray a spectrum computed without the MgH molecular features. 

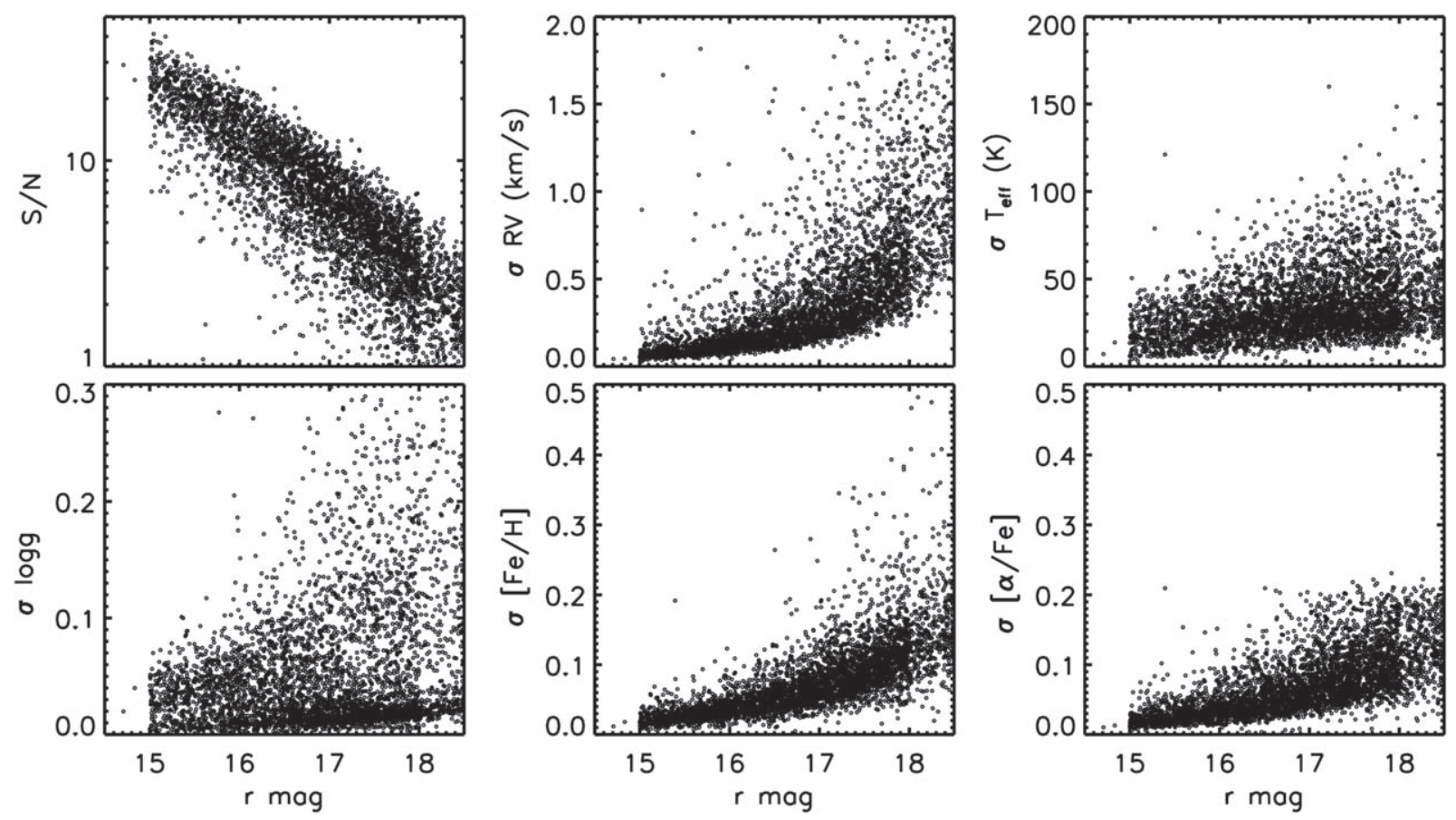

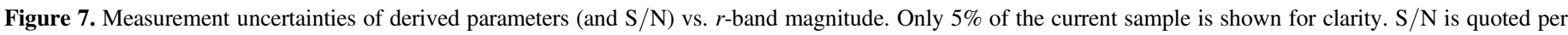
pixel. The two sequences seen in the log $g$ panel reflect the different uncertainties obtained for dwarfs vs. giants (the former being smaller than the latter).

1970). Both atmospheres and spectra are computed in 1D assuming plane-parallel geometry and LTE. We adopt the solar abundances from Asplund et al. (2009), which is also the abundance scale used in the MIST isochrones. For the spectral synthesis, a constant microturbulence of $v_{t}=1 \mathrm{~km} \mathrm{~s}^{-1}$ is adopted for all spectra. The spectral grids include variable $[\alpha / \mathrm{Fe}]$ abundances, though we note that the isochrone tables do not currently include this level of flexibility. Atomic and molecular line lists are adopted from the latest compilation of R. Kurucz, and have been astrophysically calibrated against ultra high resolution spectra of the Sun and Arcturus using the same model assumptions as adopted herein.

In addition to the mass, age, and metallicity that determine the location along an isochrone, MINESweeper also determines the distance, reddening $\left(A_{\mathrm{V}}\right),[\alpha / \mathrm{Fe}], \mathrm{RV}$, stellar broadening (representing both rotational broadening and macroturbulence), instrumental resolution, and four Chebyshev coefficients for matching the spectral shape between the data and model. Recall that the initial preparation of the data includes a continuum normalization procedure based on the twilight exposures. Experimentation led us to settle on a fourth-order Chebyshev polynomial for fine-tuning the continuum matching between the data and model. The total number of free parameters being estimated from the data is therefore 13.

Each free parameter requires a prior. Since we are focused on the stellar halo, which is comprised of old stars, we adopt a uniform log age prior of $[10.0,10.15] \log (\mathrm{yr})$. This is consistent with the approach taken by many other authors (e.g., Burnett \& Binney 2010; Schönrich \& Bergemann 2014; Xue et al. 2014). A Kroupa (2001) IMF is adopted for the mass prior. A firstpass estimate of the RV is determined by a simple crosscorrelation using a template with $T_{\text {eff }}$ determined from a firstpass fit to the SED and fixed values of $[\mathrm{Fe} / \mathrm{H}]=-1.0,[\alpha /$ $\mathrm{Fe}]=0.0$, and $\log g=2.0$. The prior on $\mathrm{RV}$ in the main program is taken to be uniform centered on the initial guess with a width of $\pm 25 \mathrm{~km} \mathrm{~s}^{-1}$. The reddening prior is a Gaussian centered on the Schlegel et al. (1998) dust map value with a width of $15 \%$ (we have adopted the $14 \%$ lower normalization of the dust maps provided by Schlafly \& Finkbeiner 2011). The prior on metallicity is uniform in the range $[-4.0,0.5]$, and $[\alpha /$ $\mathrm{Fe}]$ from $[-0.2,0.6]$. Rotational broadening is restricted to $[0$, 15] $\mathrm{km} \mathrm{s}^{-1}$ with an additional Gaussian prior centered on zero with a width of $3 \mathrm{~km} \mathrm{~s}^{-1}$. The prior on the instrumental resolution is a Gaussian centered on $R=25,000$ with a standard deviation of 1000. Gaia DR2 parallaxes are used as a Gaussian prior, which aides in the separation between dwarf and giant solutions even when the Gaia parallax has low $\mathrm{S} / \mathrm{N}$. We adopt a Gaia parallax zero-point offset of +0.05 , consistent with recent literature estimates (e.g., Leung \& Bovy 2019; Schönrich et al. 2019). Sampling is performed in log distance, which is equivalent to assuming a prior on the stellar density profile of $n \propto d^{-3}$. See Bailer-Jones et al. (2018) for an example of a more complex set of priors for determining distances. Finally, we note that the isochrones are restricted to evolutionary points between the zero age main sequence and the beginning of the first thermal pulse.

MINESweeper enables measurements of metallicities to values as low as -4.0 , although we caution that estimates below $\approx-2.3$ have not been validated against literature estimates (see the next section). The current setup enables measurement only of $[\mathrm{Fe} / \mathrm{H}]$ and $[\alpha / \mathrm{Fe}]$. However, in the wavelength range of the survey there are atomic and molecular transitions from elements including $\mathrm{C}, \mathrm{Na}, \mathrm{Mg}, \mathrm{Ca}, \mathrm{Ti}, \mathrm{V}, \mathrm{Cr}$, $\mathrm{Mn}, \mathrm{Fe}, \mathrm{Co}, \mathrm{Ni}, \mathrm{Cu}, \mathrm{Y}, \mathrm{Ce}$, and $\mathrm{Nd}$. Many of these transitions are relatively weak (line depths of a few percent) and so will be impossible to measure for the majority of the low SNR spectra in our sample. However, for the bright subset, we intend to apply complementary techniques (Ting et al. 2019) in order to measure at least some of these valuable element abundances.

The 13 free parameters are fit to the data using the dynesty nested sampling package (Speagle 2019). MINESweeper 

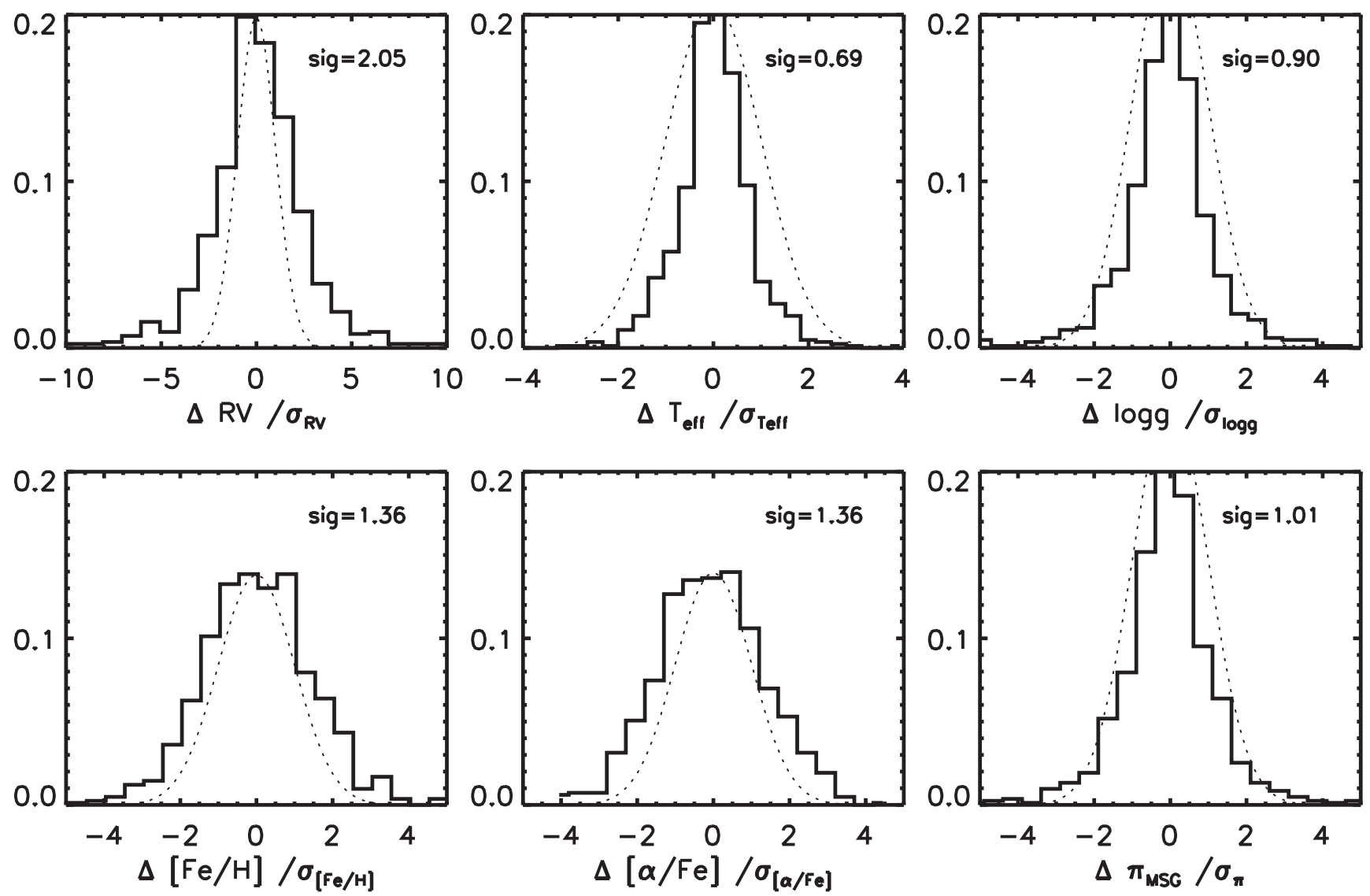

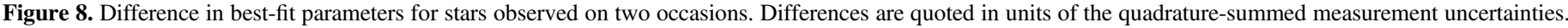

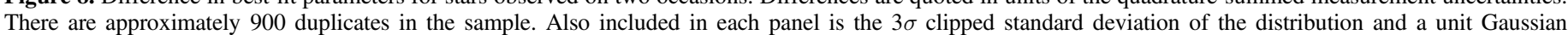

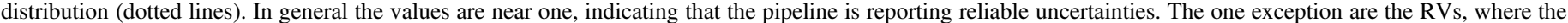
formal uncertainties appear to be underestimated by a factor of $\approx 2$ (note that the median formal uncertainty on the $\mathrm{RVs}$ is $0.24 \mathrm{~km} \mathrm{~s}{ }^{-1}$ ).

currently takes 1-2 CPU hr per star to fully sample the posterior space.

\subsubsection{Pipeline Validation}

At the beginning of each observing night twilight spectra are obtained to aid in the data reduction. We have fit all of these spectra with MINESweeper in order to test the absolute accuracy of the RVs (the twilight spectra are dominated by solar absorption lines). Taking into account the gravitational redshift and convective blueshift of the solar spectrum, MINESweeper recovers absolute velocities with an accuracy of $\pm 0.5 \mathrm{~km} \mathrm{~s}^{-1}$.

Cargile et al. (2019) fit Hectochelle spectra obtained for several open and globular clusters and showed that MINESweeper accurately recovers metallicities, $\alpha$-enhancements, distances, and locations in $\log g-T_{\text {eff }}$ space for clusters over a wide range of metallicities (from M92 at $[\mathrm{Fe} / \mathrm{H}]=-2.3$ to $\mathrm{M} 67$ at $[\mathrm{Fe} / \mathrm{H}]=0.0$ ). There are modest systematic uncertainties as a function of $\log g$, at the $\approx 0.1$ dex level. Cargile et al. also show comparisons between spectrophotometric distances and Gaia parallaxes for $\mathrm{H} 3$ stars with high $\mathrm{S} / \mathrm{N}$ Gaia parallaxes. For this comparison these authors did not use the Gaia parallaxes as a prior in the fitting. They found very good agreement with Gaia parallaxes.

Figure 7 shows the distribution of $\mathrm{S} / \mathrm{N}$ and uncertainties in $\mathrm{RV}, T_{\text {eff }}, \log g,[\mathrm{Fe} / \mathrm{H}]$, and $[\alpha / \mathrm{Fe}]$ as a function of $r$-band magnitude. We show here only $5 \%$ of the current sample for clarity. At the faint end of the primary sample $(r=18)$, the RV uncertainties are $<1 \mathrm{~km} \mathrm{~s}^{-1}$ and abundances are precise to $\approx 0.1 \mathrm{dex}$. Temperatures are measured to $\approx 50 \mathrm{~K}$. This level of precision is comparable to standard color $-T_{\text {eff }}$ relations (e.g., Casagrande et al. 2010). The $\log g$ uncertainties display two sequences, one for dwarfs (with smaller uncertainties) and the other for giants (with larger uncertainties). This is a natural consequence of the behavior of isochrones in $T_{\text {eff }}-\log g$ space: a given measurement precision in $T_{\text {eff }}$ corresponds to a much narrower range of allowed $\log g$ values on the main sequence compared to the giant branch.

In the course of the survey 12 fields were observed twice, once with the color-based selection, and once with the parallaxbased selection. This allowed us to assess the impact of the target selection (e.g., Figure 5). As a consequence of this duplication, $\approx 1000$ stars were observed twice, which provides an opportunity to test the fidelity of the stellar parameter pipeline. Figure 8 shows the difference in best-fit parameters for the duplicate observations, plotted in units of the parameter uncertainties. In general the differences follow a Gaussian with a $3 \sigma$ clipped standard deviation close to one. This means that the pipeline uncertainties are reliable.

The panel comparing RVs deserves further comment. First, the pipeline uncertainties appear to be underestimated by a factor of two. This is of little concern for our science applications, as the quoted uncertainties are very small (the median uncertainty is $0.24 \mathrm{~km} \mathrm{~s}^{-1}$; see Figure 7). As noted earlier, analysis of the twilight spectra indicates an absolute uncertainty floor of $\approx 0.5 \mathrm{~km} \mathrm{~s}^{-1}$ in the RVs. Adding this uncertainty floor in quadrature to the statistical uncertainties results in a total error 



Figure 9. Fractional distance uncertainties vs. S/N. Median values are shown as large open symbols. Left panel: results for giants (log $g<4.0$ ), color-coded by metallicity. At fixed S/N, the fraction distance uncertainties are larger at lower metallicity. Right panel: results for dwarfs (log $g>4.0$ ), color-coded by log $g$.

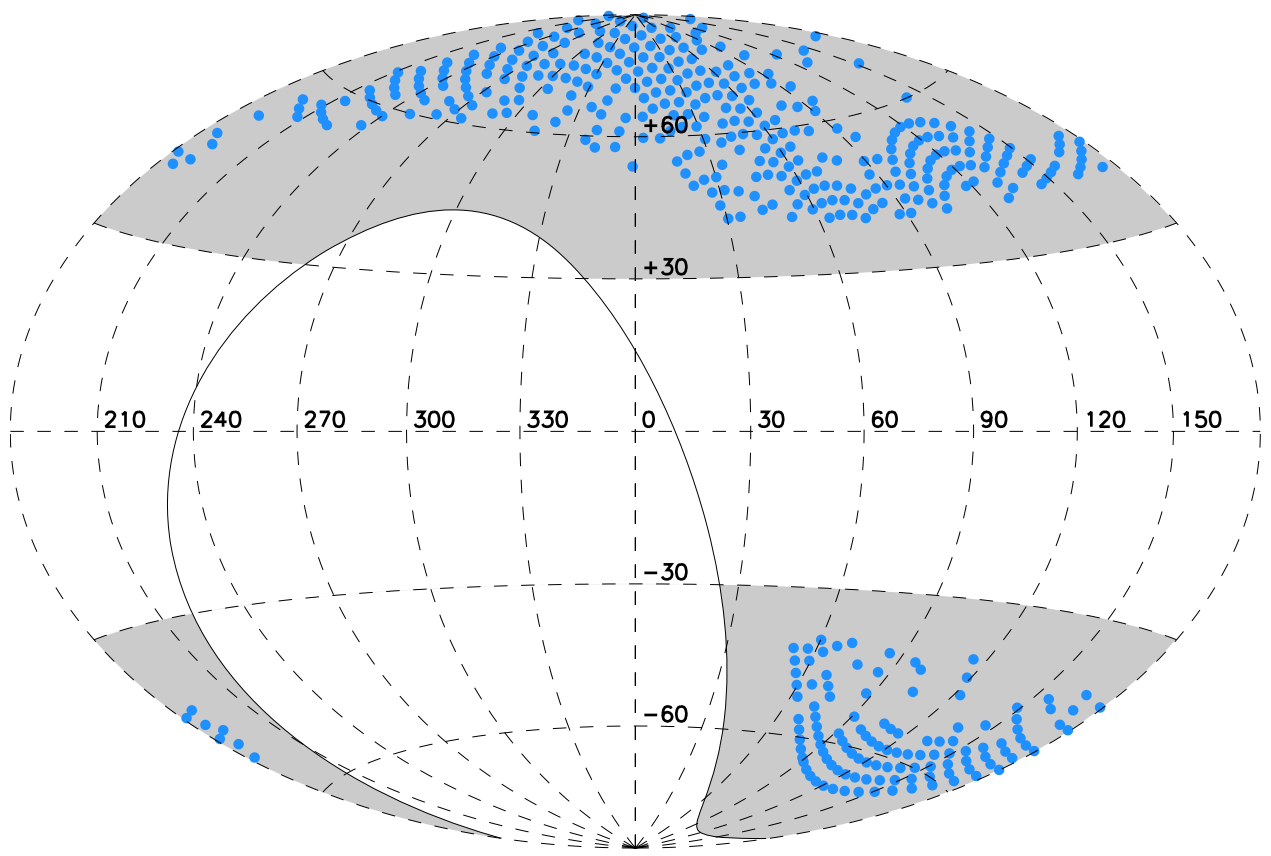

Figure 10. Survey footprint (gray) in Galactic coordinates. Fields observed as of 2019 June are shown as blue symbols (not drawn to scale). The solid line traces decl. $=-20^{\circ}$

budget in good agreement with the repeat observations. Second, there is a small but prominent population of outliers. It appears that many of these outliers are due to binarity. In some cases a second pair of lines is clearly visible in the spectrum, while in other cases the lines appear significantly broadened (e.g., by $>5 \mathrm{~km} \mathrm{~s}^{-1}$ ). These outliers will provide a valuable probe of the impact of binarity on our single-epoch RV estimates.

Figure 9 shows the fractional distance uncertainties derived from MINESweeper. Results are shown separately for giants and dwarfs. In each panel the results are color-coded by the variable that most strongly correlates with the distance uncertainties at fixed
$\mathrm{S} / \mathrm{N}([\mathrm{Fe} / \mathrm{H}]$ for the giants and $\log g$ for the dwarfs). For the dwarfs, the median uncertainties are $5 \%$ for $\mathrm{S} / \mathrm{N}>2$, while for the giants the uncertainties are $8 \%$ for $\mathrm{S} / \mathrm{N}>2$. These uncertainties are broadly consistent with previous work (e.g., Xue et al. 2014; Wang et al. 2016). See Cargile et al. for further discussion of the MINESweeper distance estimates.

\subsubsection{Areas for Improvement}

The current pipeline performs very well, as detailed in Cargile et al. and summarized above. Nonetheless, there are 


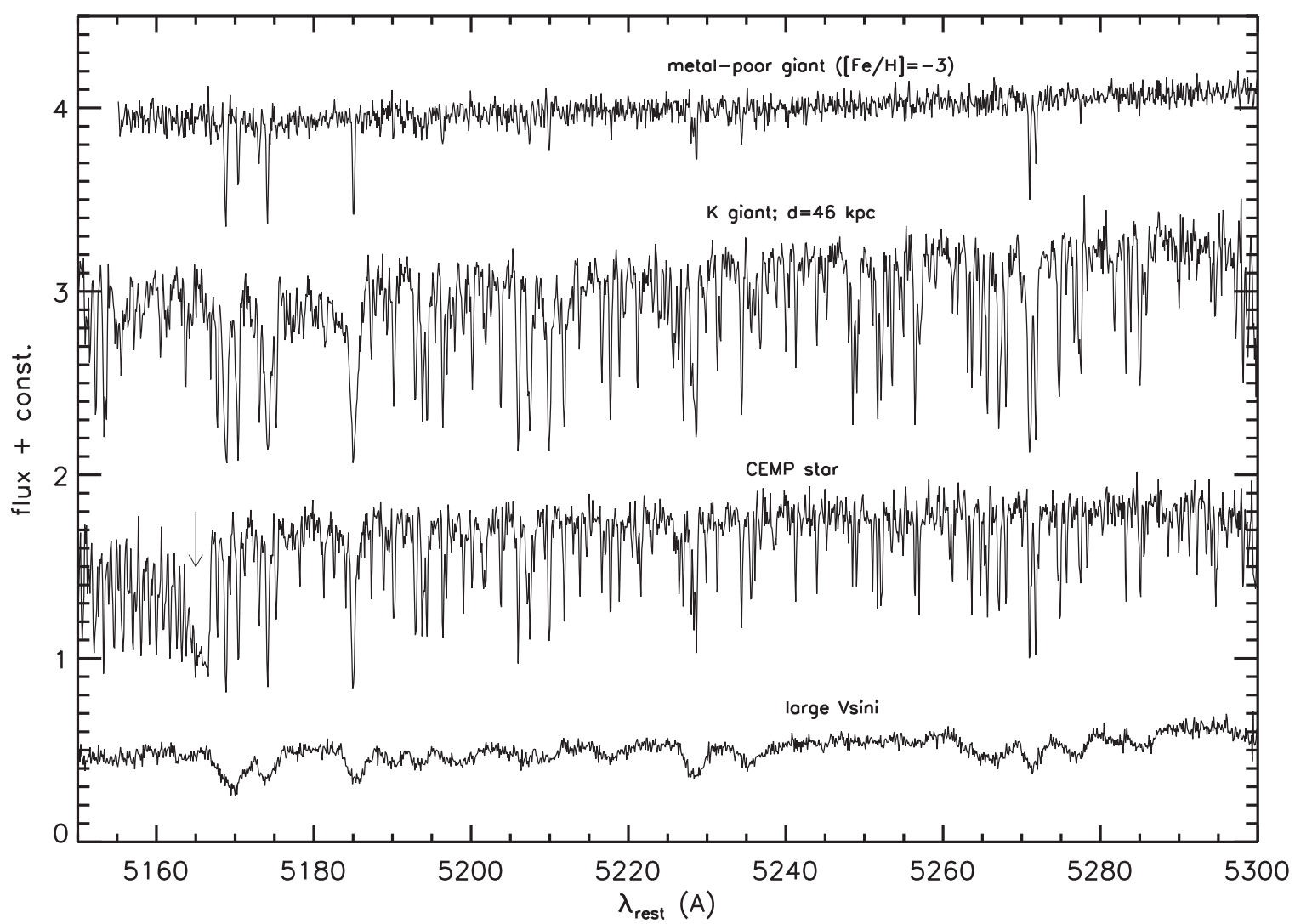

Figure 11. Several interesting and unusual spectra. From top to bottom: a metal-poor giant with $[\mathrm{Fe} / \mathrm{H}]=-3.0, \log g=1.5$, and a distance of $25 \mathrm{kpc}$; a star selected with our "K-giant" color cuts at a distance of $46 \mathrm{kpc}$ and $[\mathrm{Fe} / \mathrm{H}]=-1.0$; a carbon-enhanced giant at a preliminary distance of $19 \mathrm{kpc}$ (the $\mathrm{C}_{2}$ molecular feature is indicated with an arrow); a star with large projected rotation velocity. The $\mathrm{S} / \mathrm{N}$ of these spectra are $\gtrsim 20$.
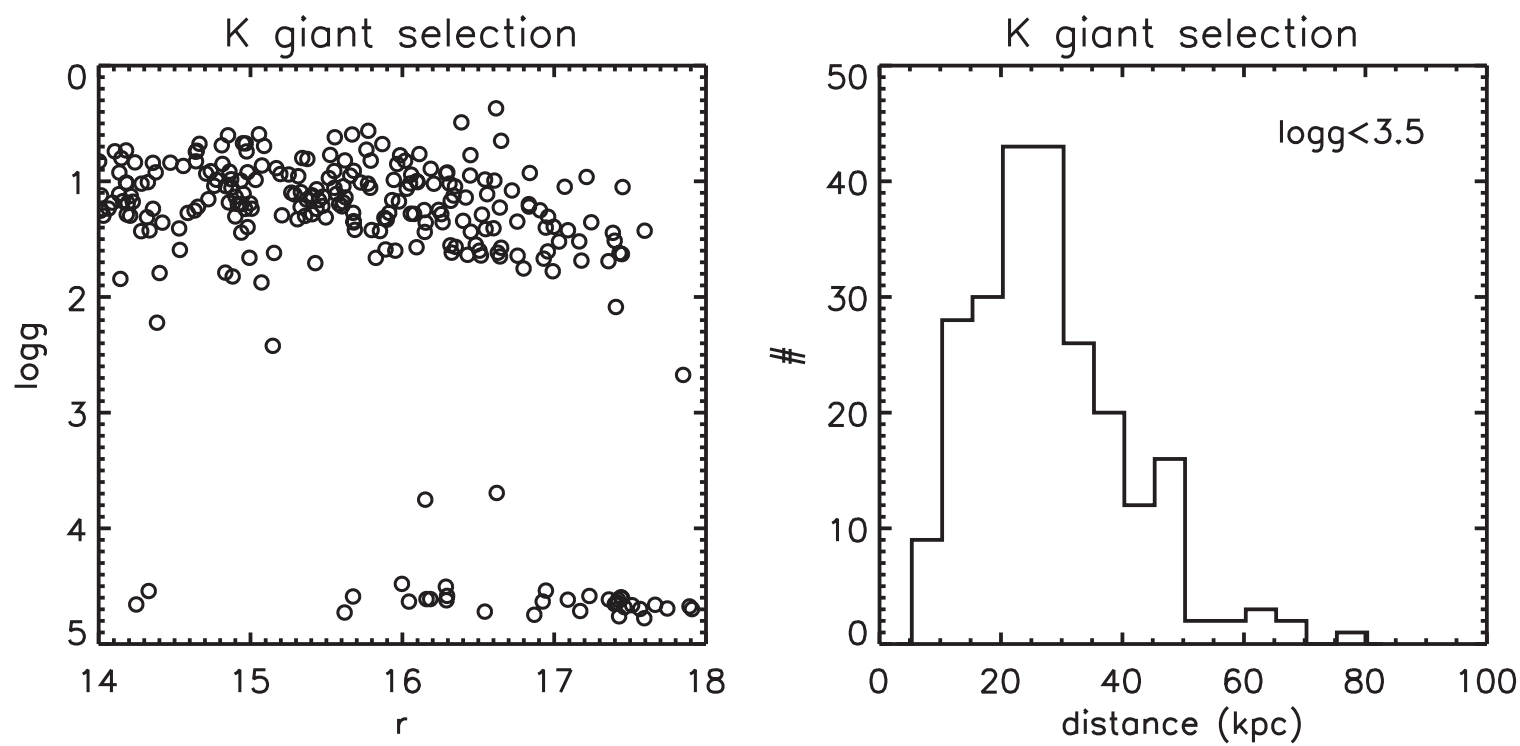

Figure 12. Validation of the photometric K-giant selection from Conroy et al. (2018). Left panel: $\log g$ vs. $r$-band magnitude for the 272 photometrically selected $\mathrm{K}$-giants observed to-date with $\mathrm{S} / \mathrm{N}>2$. Giants comprise $87 \%$ of the sample. Right panel: distribution of distances for the giants. The median distance is $24 \mathrm{kpc}$ with the most distant star at $79 \mathrm{kpc}$.

several areas where improvement is warranted. First, the current isochrone tables do not allow for variation in $[\alpha / \mathrm{Fe}]$. This limitation is currently being addressed within the MIST collaboration. Second, the lower main sequence of standard stellar models does not reproduce the observations (e.g., Feiden \& Chaboyer 2012; Choi et al. 2016). The reason for this discrepancy is believed to be related to magnetic fields, which standard stellar models do not include. We are currently working on a simple and empirical starspot model to add on top of the MIST isochrones, which will by construction result in better agreement with observations at the lower main sequence. We are also considering more informative priors on the distances (as in, e.g., Bailer-Jones et al. 2018). 
Stellar binarity is an important source of systematic uncertainty in the current pipeline. Binarity will influence our data in two possible ways: as double-lined spectroscopic binaries, and as unresolved photometric binaries. Both will bias the derived stellar parameters in systematic ways, though we expect this effect to be limited to dwarfs, as giants will greatly outshine a dwarf companion. We are developing techniques for identifying binaries, but this will remain a challenge especially when the Gaia parallaxes are of low $\mathrm{S} / \mathrm{N}$ and there is no obvious spectroscopic signature of a binary.

On the spectral modeling side, we plan to revisit the astrophysical calibration of the line list used to generate the synthetic spectra. In previous work the line list was tuned only to the Sun and Arcturus. By adding several additional calibrators (e.g., Barnard's star) we hope to achieve higher accuracy for the coolest stars. Finally, we have fixed the microturbulent velocity, $v_{t}$, to $1 \mathrm{~km} \mathrm{~s}^{-1}$. However, this parameter is known to vary with stellar type and evolutionary phase (e.g., Ramírez et al. 2013), so in subsequent work we intend to expand the spectral grid so that $v_{t}$ can be a fitted parameter.

\section{Progress To-date}

The survey began collecting data in the fall of 2017. As of 2019 June we have observed 469 fields and collected 87,930 spectra of 86,598 stars. The locations of the observed fields in Galactic coordinates are shown in Figure 10.

In the remainder of this section we provide a brief overview of the survey data.

Figure 11 shows a gallery of several interesting and unusual spectra from the current sample. The spectra include (from top to bottom) a metal-poor giant with $[\mathrm{Fe} / \mathrm{H}]=-3.0, \log g=1.5$, and a distance of $25 \mathrm{kpc}$; a star selected with our "K-giant" color cuts at a distance of $46 \mathrm{kpc}$; a carbon-enhanced giant at a preliminary distance of $19 \mathrm{kpc}$ (the $\mathrm{C}_{2}$ molecular feature is indicated with an arrow). CEMP stars are known to be common at low metallicity, with an estimated frequency $>10 \%$ at $[\mathrm{Fe} /$ $\mathrm{H}]<-2$ (Lee et al. 2013).

The last spectrum in Figure 11 is a star with a large projected rotation velocity. While unusual, this type of star is not unique in our survey: there are several hundred stars that have pipeline-based rotation velocities that are limited by the imposed prior of $15 \mathrm{~km} \mathrm{~s}^{-1}$. By-eye inspection of these stars indicates that many have much higher rotational velocities. Some of these stars have high S/N Gaia parallaxes and so can be placed on an absolute-magnitude CMD. Doing so reveals that most of these stars reside on or near the equal mass binary star sequence. It will be interesting to follow up these stars in detail as a unique probe of the binary star population in the stellar halo.

As discussed in Section 2.2, we have included in our target selection the photometrically identified $\mathrm{K}$ giants from Conroy et al. (2018). These stars are rare but of potentially high value as they can be seen to great distances $(>100 \mathrm{kpc})$. In Figure 12 we show the spectroscopically confirmed $\log g$ values for this sample as a function of $r$-band magnitude. The measured $\log g$ values confirm the results presented in Conroy et al. (2018) that the photometric $\mathrm{K}$ giant selection is highly pure-in our survey $87 \%$ of these stars are bona fide giants. The purity decreases at fainter magnitudes, which we believe is due to increasing photometric scatter in the WISE photometry used in the selection criteria. The right panel shows the distribution of

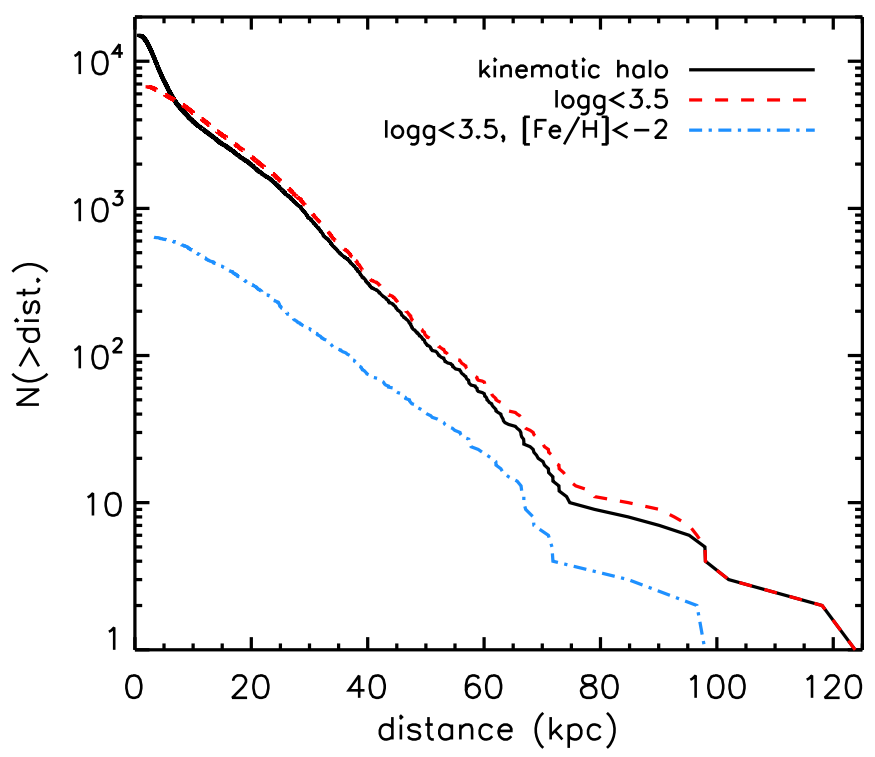

Figure 13. Cumulative histogram of heliocentric distances to stars in the H3 survey. Spectrophotometric distances are estimated via the MINESweeper program. We show the distribution of kinematically selected halo stars (solid line), giants ( $\log g<3.5$; dashed line), and low metallicity giants ( $\log g<3.5$ and $[\mathrm{Fe} / \mathrm{H}]<-2$; dotted-dashed line). Results are shown for data collected as of 2019 June.

distances of these stars; the mean distance of $24 \mathrm{kpc}$ and the most distant star is at $79 \mathrm{kpc}$.

Finally, Figure 13 shows the distribution of heliocentric distances of kinematically defined halo stars, giants, and metalpoor giants. Note that this is simply the cumulative count distribution and does not include a correction for the selection function. An estimation of the halo density profile from these data will be the subject of future work.

\section{5. $\mathrm{H3}$ in Context}

There are multiple recently completed, ongoing, and planned large-scale ground-based stellar spectroscopic surveys. In this section we place H3 in context with these other surveys.

Figure 14 compares a number of large area medium resolution $(R>8000)$ spectroscopic surveys. The left panel shows the total number of stars from each survey as a function of the limiting $G$-band magnitude. The surveys include the completed RAVE (Steinmetz et al. 2006) and Gaia-ESO (Gilmore et al. 2012) surveys, the ongoing H3, APOGEE (Majewski et al. 2017) and GALAH (Martell et al. 2017) surveys, and the upcoming SDSS-V (Kollmeier et al. 2017), WEAVE (Dalton et al. 2012), and 4MOST (Guiglion et al. 2019) surveys. In the case of ongoing or planned surveys we plot their quoted final number of expected targets. Most surveys have limiting magnitudes in filters other than the $G$-band (e.g., APOGEE and SDSS-V are $H$-band selected), so the translation to a limiting $G$-band magnitude is approximate. The Gaia $G$-band is close to the SDSS and Pan-STARRS $r$-band (to within $\lesssim 0.05 \mathrm{mag}$ ) for $4000<T_{\text {eff }}<6500 \mathrm{~K}$.

We have not included low resolution stellar spectroscopic surveys, such as the completed SEGUE survey (Yanny et al. 2009), the ongoing LAMOST survey (Deng et al. 2012), and the upcoming DESI survey (DESI Collaboration et al. 2016). Moreover, while both of the upcoming WEAVE and 4MOST surveys have medium and low resolution components; in Figure 14 we only include the medium resolution surveys. 

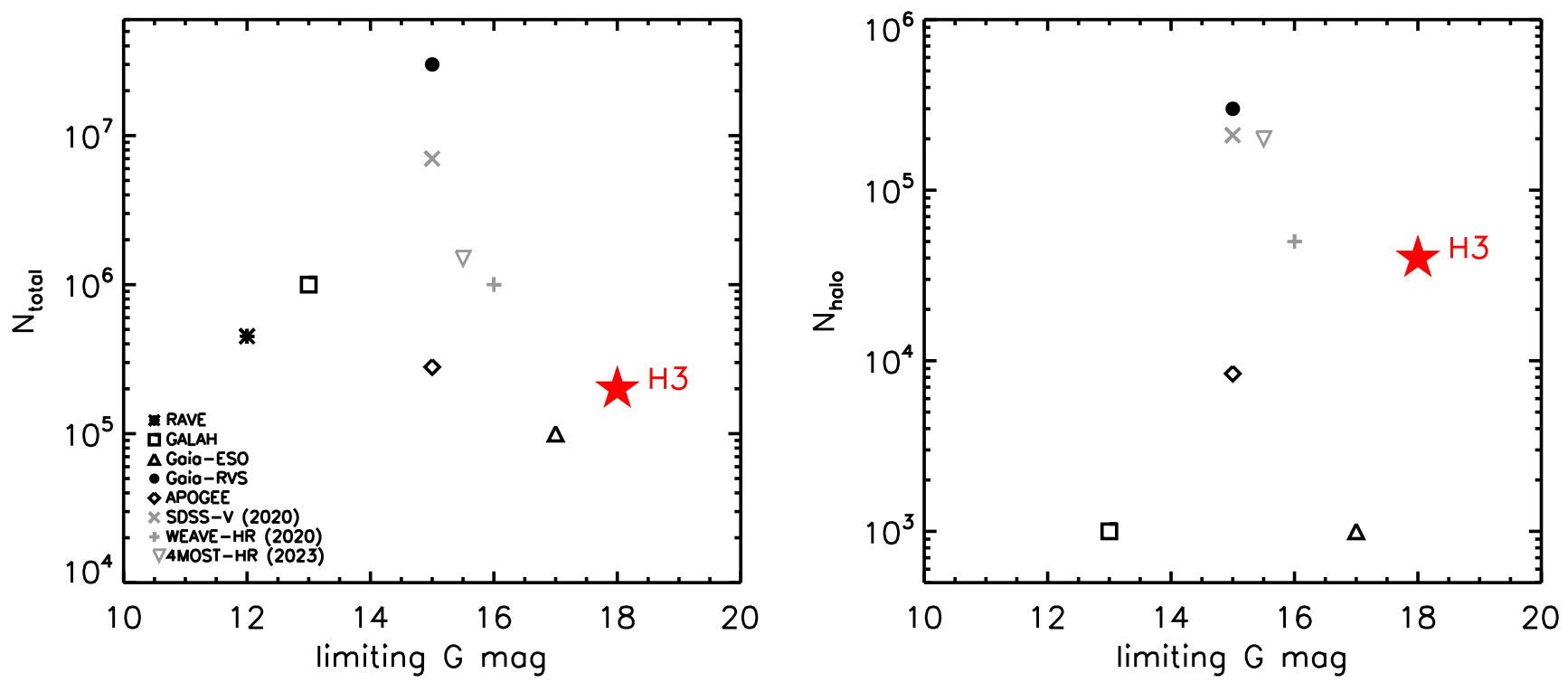

Figure 14. Comparison of existing and planned stellar spectroscopic surveys with a spectral resolution of $R>8000$. Left panel: total number of stars expected as a function of the limiting $G$-band magnitude. Right panel: estimated number of halo stars from each survey (see the text for details). Ongoing or completed surveys are shown as black points, while upcoming surveys are shown as gray (along with anticipated survey start dates in parentheses).

We caution that it is not obvious whether medium or low resolution is "better" in the context of a fixed survey speed (e.g., number of stars per night) and measurement precision requirements. As argued in Ting et al. (2017), holding the exposure time and the number of pixels per spectrum fixed, the theoretical information content is nearly independent of spectral resolution. Of course, at low spectral resolution one has to contend with a greater variety of "subresolution" effects, including line blending. For example, $R=2000$ corresponds to a velocity dispersion of $63 \mathrm{~km} \mathrm{~s}^{-1}$, so velocity precision of $<5 \mathrm{~km} \mathrm{~s}^{-1}$ (likely the relevant scale for identifying substructure in phase space) requires identifying line centers at the $1 / 10$ pixel level. This is not impossible, and with sufficient calibrating data should be achievable. An argument in favor of lower resolution is access to a larger number of transitions from more species than would be available from a high resolution spectrum, if the total number of pixels is held fixed.

Another important distinguishing feature in the landscape of surveys is the hemispheric coverage. Surveys covering the northern hemisphere are WEAVE, LAMOST, DESI, and H3. Surveys covering the south are RAVE, GALAH, Gaia-ESO, and 4MOST. All-sky surveys include Gaia, APOGEE, and SDSS-V (the latter two have twin spectrographs operating at northern and southern observatories).

It is challenging to provide comparisons across surveys with such a wide variety of survey strategies. In addition to depth, number of stars, and spectral resolution, one must also consider exposure time and hence $\mathrm{S} / \mathrm{N}$, wavelength coverage, targeting strategy, and on-sky footprint (e.g., northern versus southern hemispheres, disk versus halo fields, sparse versus dense tiling). Comparisons such as those shown in Figure 14 should therefore be interpreted with these considerations in mind.

The right panel of Figure 14 shows an estimate of the number of halo stars that each survey has or will yield. To construct this plot we have assumed the following halo fractions: $0.1 \%$ for GALAH (from Martell et al. 2017), $1 \%$ for Gaia-RVS (estimated from the Rybizki et al. 2018, mock catalog), 1\% for Gaia-ESO Survey (G. Gilmore 2019, private communication), 3\% for APOGEE and SDSS-V (this is the kinematic halo fraction estimated from an APOGEE-Gaia cross-matched catalog; we adopt the same fraction for SDSS$\mathrm{V})$, and $20 \%$ for H3. The numbers for WEAVE-HR are from S. Jin et al. (2019, in preparation), and the 4MOST-HR halo population is from Christlieb et al. (2019). The halo fraction in H3 is an order of magnitude higher than in any other moderateresolution survey. Among the surveys with an appreciable number of halo stars, $\mathrm{H} 3$ is two magnitudes deeper. For a given stellar type (e.g., TRGB, red clump), this increases the maximum observable distance by a factor of 2.5 .

In Figure 15 we provide a more detailed comparison between three surveys: H3, APOGEE, and LAMOST. In the left panel we show histograms of metallicity for the entire sample from each survey, subject to a few quality cuts (H3: S/ $\mathrm{N}>2$ and quality flag $=0$; APOGEE: ASPCAPFLAG $=0$; LAMOST: $\mathrm{S} / \mathrm{N}_{g}>40$, and objtype = "star"). LAMOST clearly dominates the overall sample. Notice that $\mathrm{H} 3$ has more low metallicity $([\mathrm{Fe} / \mathrm{H}]<-1)$ stars than APOGEE. For APOGEE and LAMOST the metallicities are truncated at -2.5 , which is an artifact of the spectral grids used in estimating stellar parameters. Moreover, while the $\mathrm{H} 3$ metallicities have been well-tested down to -2.5 , we caution that the lowest metallicities will require careful vetting.

The right panel of Figure 15 shows the same histograms of metallicity with the additional requirements that $|b|>30^{\circ}$ and $\pi-2 \sigma_{\pi}<0.5$ mas. This is the selection function for $\mathrm{H} 3$, so it is not too surprising that with these restrictions $\mathrm{H} 3$ contains more metal-poor stars even than LAMOST. Nonetheless, this plot highlights the strengths of the $\mathrm{H} 3$ survey in comparison to existing large spectroscopic surveys. Comparison between the left and right figures highlights the fact that while $\mathrm{H} 3$ is not the largest spectroscopic survey, it is by far the largest medium resolution survey of distant high latitude stars, and rivals low resolution surveys of such populations. 

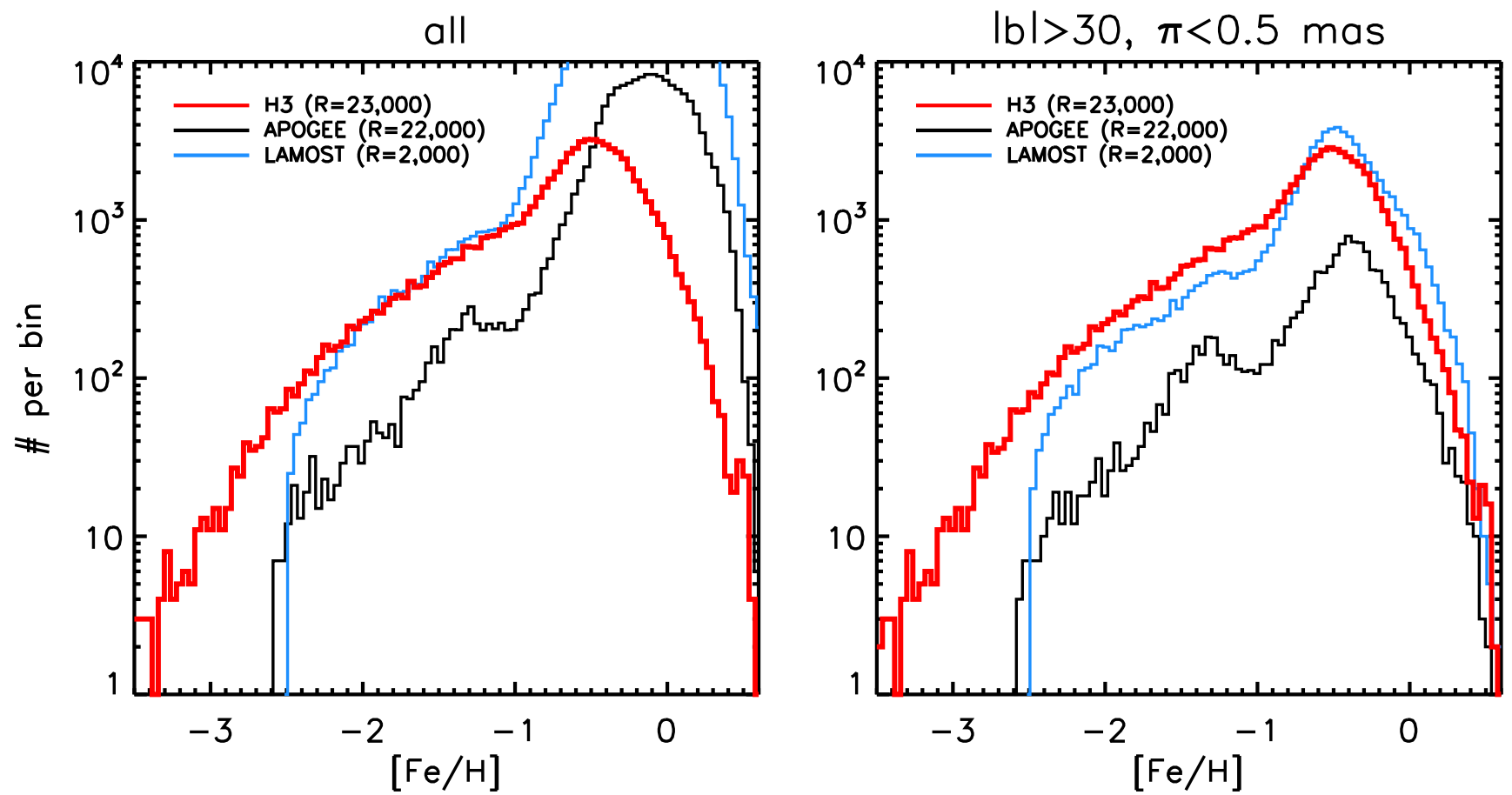

Figure 15. Left panel: metallicity distribution of the current H3 Survey compared to the APOGEE and LAMOST Surveys. Right panel: metallicity distributions for the three surveys for all stars with Galactic latitude $|b|>30^{\circ}$ and a Gaia DR2 parallax $\pi<0.5$ mas. H3 stars were selected according to these criteria, whereas the other two surveys were not. Comparison between the left and right panels highlights the fact that while $\mathrm{H} 3$ is not the largest spectroscopic survey, it is by far the largest medium resolution survey of distant high latitude stars, and rivals low resolution surveys of such populations. Histograms were computed with a bin size of 0.04 dex.

Table 1

H3 at a Glance

\begin{tabular}{lc}
\hline \hline Telescope & MMT (6.5 m; Mt Hopkins, AZ) \\
Instrument & Hectochelle \\
Spectral resolution & $R=23,000$ \\
Wavelength range & $5150-5300 \AA$ \\
Number of stars & $N=200,000$ \\
Time-frame & $2017-2021$ \\
Magnitude range & $15<r<18$ \\
Main sample selection & $\pi<0.5$ mas \\
Footprint & $|b|>30^{\circ} ;$ decl. $>-20^{\circ}$ \\
Median S/N & 6 per pixel \\
Kinematic halo fraction & $\approx 20 \%$ \\
&
\end{tabular}

\section{Summary}

We have described a new stellar spectroscopic survey targeting the stellar halo. The goal is to trace the assembly history of the Galaxy by studying the distribution of stars in $6 \mathrm{D}$ phase space plus chemistry. The survey aims to collect 200,000 stars sparsely sampled over 15,000 sq. degrees. The target selection is deliberately simple and interpretable: a magnitude range of $15<r<18$ and Gaia DR2 parallaxes such that $\pi-2 \sigma_{\pi}<0.5$ mas (recently updated to $\pi<0.4$ mas). As of 2019 June we have collected 88,000 spectra. The MINESweeper stellar parameter pipeline is delivering $\mathrm{RVs},[\mathrm{Fe} / \mathrm{H}]$, $[\alpha / \mathrm{Fe}]$, and spectrophotometric distances for every star. A summary of the key parameters of the survey is provided in Table 1. All of the data, including the derived stellar parameters, will eventually be made publicly available via the survey website: h3survey.rc.fas.harvard.edu.

We thank the CfA and U. Arizona TACs for their continued support of this long-term program. Observations reported here were obtained at the MMT Observatory, a joint facility of the Smithsonian Institution and the University of Arizona. This research was supported in part by the National Science Foundation under grant No. NSF PHY-1748958. C.C. acknowledges the hospitality of the KITP, where this paper was written. The computations in this paper were run on the Odyssey cluster supported by the FAS Division of Science, Research Computing Group at Harvard University.

\section{ORCID iDs}

Charlie Conroy (ib https://orcid.org/0000-0002-1590-8551

Ana Bonaca (10) https://orcid.org/0000-0002-7846-9787

Nelson Caldwell (1) https://orcid.org/0000-0003-2352-3202

Dennis Zaritsky (1) https://orcid.org/0000-0002-5177-727X

\section{References}

Asplund, M., Grevesse, N., Sauval, A. J., \& Scott, P. 2009, ARA\&A, 47, 481 Bagnulo, S., Jehin, E., Ledoux, C., et al. 2003, Msngr, 114, 10

Bailer-Jones, C. A. L., Rybizki, J., Fouesneau, M., Mantelet, G., \& Andrae, R. 2018, AJ, 156, 58

Belokurov, V., Deason, A. J., Erkal, D., et al. 2019, MNRAS, 488, L47

Belokurov, V., Erkal, D., Evans, N. W., Koposov, S. E., \& Deason, A. J. 2018, MNRAS, 478, 611

Bonaca, A., Conroy, C., Wetzel, A., Hopkins, P. F., \& Kereš, D. 2017, ApJ, 845,101

Bullock, J. S., \& Boylan-Kolchin, M. 2017, ARA\&A, 55, 343

Bullock, J. S., \& Johnston, K. V. 2005, ApJ, 635, 931

Burnett, B., \& Binney, J. 2010, MNRAS, 407, 339

Cargile, P., Conroy, C., Johnson, B., et al. 2019, ApJ, submitted (arXiv:1907. 07690)

Casagrande, L., Ramírez, I., Meléndez, J., Bessell, M., \& Asplund, M. 2010, A\&A, 512, A54

Chambers, K. C., Magnier, E. A., Metcalfe, N., et al. 2016, arXiv:1612.05560

Choi, J., Dotter, A., Conroy, C., et al. 2016, ApJ, 823, 102

Christlieb, N., Battistini, C., Bonifacio, P., et al. 2019, Msngr, 175, 26

Conroy, C., Bonaca, A., Naidu, R. P., et al. 2018, ApJL, 861, L16 
Cooper, A. P., Cole, S., Frenk, C. S., et al. 2010, MNRAS, 406, 744 Cutri, R. M. 2013, yCat, 2328

Dalton, G., Trager, S. C., Abrams, D. C., et al. 2012, Proc. SPIE, 8446, 84460P

Deason, A. J., Belokurov, V., Evans, N. W., \& Johnston, K. V. 2013, ApJ, 763,113

Deason, A. J., Belokurov, V., Koposov, S. E., \& Rockosi, C. M. 2014, ApJ, 787, 30

Deng, L.-C., Newberg, H. J., Liu, C., et al. 2012, RAA, 12, 735

DESI Collaboration, Aghamousa, A., Aguilar, J., et al. 2016, arXiv:1611. 00036

Fabricant, D., Fata, R., Roll, J., et al. 2005, PASP, 117, 1411

Fattahi, A., Belokurov, V., Deason, A. J., et al. 2019, MNRAS, 484, 4471

Feiden, G. A., \& Chaboyer, B. 2012, ApJ, 757, 42

Frebel, A., \& Norris, J. E. 2015, ARA\&A, 53, 631

Gaia Collaboration, Brown, A. G. A., Vallenari, A., et al. 2018, A\&A, 616, A1

Garavito-Camargo, N., Besla, G., Laporte, C. F. P., et al. 2019, arXiv:1902.05089

Gilmore, G., Randich, S., Asplund, M., et al. 2012, Msngr, 147, 25

Gómez, F. A., Besla, G., Carpintero, D. D., et al. 2015, ApJ, 802, 128

Guiglion, G., Battistini, C., Bell, C. P. M., et al. 2019, Msngr, 175, 17

Helmi, A. 2008, A\&ARv, 15, 145

Helmi, A., Babusiaux, C., Koppelman, H. H., et al. 2018, Natur, 563, 85

Helmi, A., \& White, S. D. M. 1999, MNRAS, 307, 495

Hinkle, K., Wallace, L., Valenti, J., \& Harmer, D. 2000, Visible and Near Infrared Atlas of the Arcturus Spectrum 3727-9300 $\AA$ (San Francisco, CA: ASP)

Jean-Baptiste, I., Di Matteo, P., Haywood, M., et al. 2017, A\&A, 604, A106 Johnston, K. V., Hernquist, L., \& Bolte, M. 1996, ApJ, 465, 278

Juric, M. 2012, LSD: Large Survey Database Framework, Astrophysics Source Code Library, ascl:1209.003

Kollmeier, J. A., Zasowski, G., Rix, H.-W., et al. 2017, arXiv:1711.03234

Kroupa, P. 2001, MNRAS, 322, 231

Kurucz, R. L. 1970, SAOSR, 309
Kurucz, R. L. 1993, SYNTHE Spectrum Synthesis Programs and Line Data (Cambridge, MA: Smithsonian Astrophysical Observatory)

Lee, Y. S., Beers, T. C., Masseron, T., et al. 2013, AJ, 146, 132

Leung, H. W., \& Bovy, J. 2019, MNRAS, 489, 2079

Lindegren, L., Hernández, J., Bombrun, A., et al. 2018, A\&A, 616, A2

Lowing, B., Wang, W., Cooper, A., et al. 2015, MNRAS, 446, 2274

Majewski, S. R., Schiavon, R. P., Frinchaboy, P. M., et al. 2017, AJ, 154, 94

Martell, S. L., Sharma, S., Buder, S., et al. 2017, MNRAS, 465, 3203

Myeong, G. C., Evans, N. W., Belokurov, V., Sanders, J. L., \& Koposov, S. E. 2018, ApJL, 856, L26

Perryman, M. A. C., de Boer, K. S., Gilmore, G., et al. 2001, A\&A, 369, 339

Ramírez, I., Allende Prieto, C., \& Lambert, D. L. 2013, ApJ, 764, 78

Rybizki, J., Demleitner, M., Fouesneau, M., et al. 2018, PASP, 130, 074101

Schlafly, E. F., \& Finkbeiner, D. P. 2011, ApJ, 737, 103

Schlegel, D. J., Finkbeiner, D. P., \& Davis, M. 1998, ApJ, 500, 525

Schönrich, R., \& Bergemann, M. 2014, MNRAS, 443, 698

Schönrich, R., McMillan, P., \& Eyer, L. 2019, MNRAS, 487, 3568

Sesar, B., Hernitschek, N., Dierickx, M. I. P., Fardal, M. A., \& Rix, H.-W. 2017, ApJL, 844, L4

Simon, J. D., \& Geha, M. 2007, ApJ, 670, 313

Speagle, J. S. 2019, arXiv:1904.02180

Stassun, K. G., \& Torres, G. 2018, ApJ, 862, 61

Steinmetz, M., Zwitter, T., Siebert, A., et al. 2006, AJ, 132, 1645

Szentgyorgyi, A., Furesz, G., Cheimets, P., et al. 2011, PASP, 123, 1188

Ting, Y.-S., Conroy, C., Rix, H.-W., \& Cargile, P. 2017, ApJ, 843, 32

Ting, Y.-S., Conroy, C., Rix, H.-W., \& Cargile, P. 2019, ApJ, 879, 69

Walker, M. G., Olszewski, E. W., \& Mateo, M. 2015, MNRAS, 448, 2717

Wang, J., Shi, J., Zhao, Y., et al. 2016, MNRAS, 456, 672

Wang, W., Han, J., Cooper, A. P., et al. 2015, MNRAS, 453, 377

Wright, E. L., Eisenhardt, P. R. M., Mainzer, A. K., et al. 2010, AJ, 140, 1868

Xue, X.-X., Ma, Z., Rix, H.-W., et al. 2014, ApJ, 784, 170

Yanny, B., Rockosi, C., Newberg, H. J., et al. 2009, AJ, 137, 4377 